\title{
A Prospective Study of Patients with Brain MRI Showing Incidental T2 Hyperintensities Addressed as Multiple Sclerosis: a Lot of Work to do Before Treating
}

Christine Lebrun • Mikael Cohen • Annabelle Chaussenot •

Lydiane Mondot $\cdot$ Stephane Chanalet

To view enhanced content go to www.neurologytherapy-open.com

Received: October 24, 2014 / Published online: December 13, 2014

(c) The Author(s) 2014. This article is published with open access at Springerlink.com

\section{ABSTRACT}

Introduction: With the development of magnetic resonance imaging (MRI) and publications about radiologically isolated syndrome (RIS), a lot of patients are referred to multiple sclerosis (MS) tertiary centers to confirm diagnosis of RIS or MS when brain T2 abnormalities are identified, whatever their characteristics. We evaluate prospectively the occurrence of RIS or MS and sensitivity, specificity and predictive value of McDonald criteria in diagnosis for patients presenting with incidental brain MRI T2 lesions.

Methods: The authors ran standardized procedures on 220 consecutive patients addressed by general practitioners or neurologists to confirm RIS or MS diagnosis on

Electronic supplementary material The online version of this article (doi:10.1007/s40120-014-0024-7) contains supplementary material, which is available to authorized users.

C. Lebrun $(\square)$

Service de Neurologie, Hôpital Pasteur, 30 voie romaine, 06002 Nice, France e-mail: lebrun.c@chu-nice.fr

M. Cohen · A. Chaussenot - L. Mondot .

S. Chanalet

Hôpital Pasteur, 30 voie romaine, Nice, France brain MRI and give a therapeutic advice. All patients underwent neurological tests, extensive blood screening, cerebrospinal fluid (CSF) examination, visual evoked potential (VEP) and follow-up MRI after 3, 6, 12 and 24 months to consider dissemination in time and space.

Results: Patient characteristics were: 165 women and 55 men, mean age: 42.7 years old (23-59). The major symptom motivating MRI was headaches (39\%), sensitive atypical manifestations or pain (12\%), mood disorders (10\%), transient visual symptoms (9\%), fatigue (8\%), hormonal screening (6\%), vertigo (6\%), cranial trauma (5\%), and dummy run for clinical study (5\%). After a structured analysis of T2 lesions, the suspected diagnosis was: inflammatory disease $45 \%$, vascular $33 \%$, nonpathological 19\%, genetic $2 \%$, and metabolic 1\%. Extensive screening confirmed the proposed diagnosis in $97 \%$ of cases. Among all the 220 proposed RIS patients, only $35.4 \%$ fulfilled the 2010 McDonald criteria, and 8\% can be categorized as RIS. Dissemination in time criteria was present for $82.7 \%$ of MS patients and $36 \%$ of RIS patients but none of the vascular or non-pathological T2 abnormalities. 
Conclusion: Even if RIS was initially suspected on MRI, only a third of the patients had an inflammatory disease. Most of the patients had either non-specific T2 lesions or a noninflammatory disease. Others were initially well categorized but had experienced clinical symptoms that could possibly be considered as a first clinical event. Overdiagnosis of MS can lead to propose an inappropriate diseasemodifying therapy.

Keywords: Brain MRI; Clinically isolated syndrome; Incidentaloma; Multiple sclerosis; Neurology; Radiologically isolated syndrome T2 hypersignals

\section{INTRODUCTION}

With the development of magnetic resonance imaging (MRI), a lot of patients are addressed to neurologists to confirm diagnosis of multiple sclerosis (MS) when brain T2 abnormalities are found, whatever their characteristics, even if white matter abnormalities are non-specific. Most of the time, the patient is presented with a radiological report notifying clearly the diagnosis of MS. A lot of non-specialized radiologists are not specifically aware of the clinico-radiological diagnosis leading to MS diagnosis. Diagnosis of MS is based on demonstrating dissemination in space (DS) and time on MRI and excluding other neurological disorders that can clinically and radiologically mimic MS [1]. The brain MRI motive has to be a seminal event, acute or progressive, suggestive of an inflammatory disease. When the patient suffers from suggestive MS symptoms, clinico-radiological criteria are helpful to diagnose a clinically isolated syndrome. McDonald criteria have been applied in clinical practice with good specificity for MRI criteria with two updates since the original publication but may require systematic screening (visual evoked potential, blood and cerebrospinal fluid analysis) [1-6]. Other criteria for diagnosis of MS have been recently proposed but are not yet validated [7]. Eventually, a lot of patients diagnosed with potential MS are not diagnosed after a second screening $[8,9]$.

For patients with non-typical MS symptoms, a systematic review of brain and spinal MRI is the major step to establish the diagnosis of a demyelinating disease. Recognition of MRI red flags, as defined by the European MAGNIMS (Magnetic Resonance Network in Multiple Sclerosis), improves diagnostic accuracy [10, $11]$.

The limited specificity of incidental nonspecific white matter abnormalities that are revealed by MRI may increase the number of misdiagnosis [10]. Even prior to the introduction of radiologically isolated syndrome (RIS) criteria, longitudinal clinical data from individuals with incidentally identified T2 lesions suggestive of multiple sclerosis (MS) were described. Healthy individuals who do not exhibit signs of neurological dysfunction commonly have brain MRI studies performed for a reason other than an evaluation for MS that reveals unexpected anomalies highly suggestive of demyelinating plaques given their size, location, and morphology. These healthy subjects lack symptomatology suggestive of MS and fulfill formal criteria for RIS, a recently described MS subtype that expands upon the phenotype of at-risk individuals for future demyelinating events. A formal description of RIS was first introduced in 2009 by Okuda et al. [12], to define this relevant cohort of individuals who are at risk for future demyelinating events. 
The authors describe here a prospective study consisting of the evaluation in clinical practice of a step-by-step procedure in patients presenting with non-specific symptoms and brain T2 hyperintensities, initially diagnosed as RIS or MS.

\section{METHODS}

From 2009 to 2012, the authors ran standardized procedures on 220 consecutive patients referred to their MS center by general practitioners (GP) or neurologists for specialized advice concerning suspected MS on brain MRI. Brain MRI was performed by the GP for nonspecific symptoms (such as headaches, atypical sensitive symptoms, blurred vision, and mood disorders).

\section{Classification of the T2 Hyperintensities}

Clinical and neurological data were reviewed with the patient and the brain MRI was analyzed by two physicians (neuroradiologists: LM, SC or 3 neurologists: CL, AC, MC) with a standardized procedure considering shape, size, number and location of $\mathrm{T} 2$ and $\mathrm{T} 1$ abnormalities on sequences with and without gadolinium (Fig. 1). Potential chronic inflammatory disease was retained if brain T2 hypersignals were $>3 \mathrm{~mm}$, ovoid and then, Barkhof and Tintore criteria were applied [2]. If more than 3 criteria were present, RIS or MS was suspected and DS was documented. If hyperintensities were suggestive but with $>3$ DS criteria, inflammatory disease involving central nervous system was suspected.

After this first step, the patients were categorized by the neurologist into a disease group with data suggestive or not of an inflammatory process. If not, another diagnosis was proposed: ischemic vascular disease, metabolic, genetic or nonpathological. Because patients were initially assessed for a radiological suspicion of MS, all available MRI criteria applicable were applied, irrespective of what the neurologist's suspected diagnosis was $[2,4,7,13,14]$.

\section{Extensive Screening to Confirm the No Better Explanation Concept}

As they want to have an answer for their diagnosis, all patients asked and agreed to undergo neurological tests, and extensive blood screening, including a complete blood count, erythrocyte sedimentation rate, serum cryoglobulins, total serum gamma globulins, serum protein immunoelectrophoresis, C-reactive protein, complement factors, angiotensin conversion enzyme, antinuclear antibodies, antinative DNA, antiphospholipid/ anticardiolipin antibodies, rheumatoid factor, antiprothrombinase, HIV, herpes simplex virus, varicella-zoster virus, hepatitis $\mathrm{C}$ and $\mathrm{B}$, syphilis, cytomegalovirus, and Epstein-Barr virus serological tests. All patients had a cerebrospinal fluid (CSF) analysis with cell count, protein level and an oligoclonal band (OCB) evaluation by isoelectrofocusing method. Detection of an OCB was considered as positive if more than 1 band that was not detected in the serum was present in the CSF.

At recruitment, patients had a VEP and a follow-up brain MRI was programmed with and without gadolinium at 3, 6 and 12 months to consider dissemination in time (DT) and DS. The MRI follow-up was standardized with Axial T1 3D: $\mathrm{TE}=1.7 ; \quad \mathrm{TR}=7.9 ; \quad \mathrm{NEX}=2 ; \quad \mathrm{FOV}$ : $260 \times 195 \mathrm{~mm} ; 1.6 \mathrm{~mm}$ with $0.8 \mathrm{~mm}$ overlap; $256 \times 192 ; 152$ slices; Axial T2/DP (FSE double écho): $\mathrm{TR}=5,000 ; \mathrm{TE}=8.0$ et $\mathrm{TE}=103.9$; $\mathrm{NEX}=2 ; \quad$ FOV: $240 \times 180 \mathrm{~mm} ; \quad 2.0 \mathrm{~mm}$; 
Patients with suspected RIS

$(N=220)$

1

Step by step analysis of brain MRI

Clinical data analysis: medical history, neurological examination
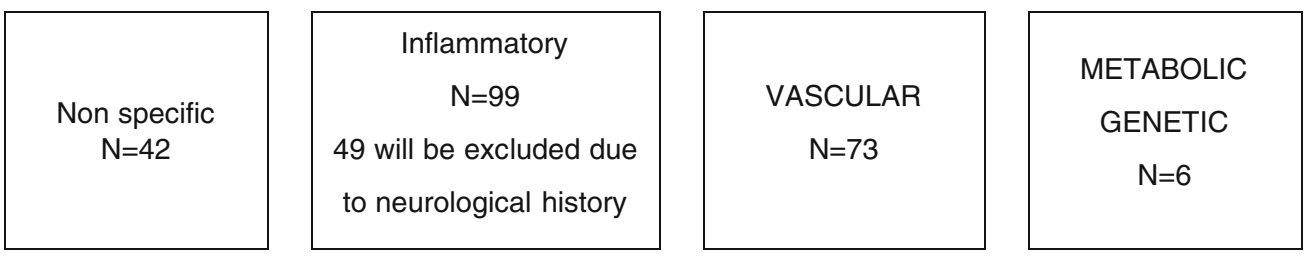

Extensive screening, blood, CSF, VEP

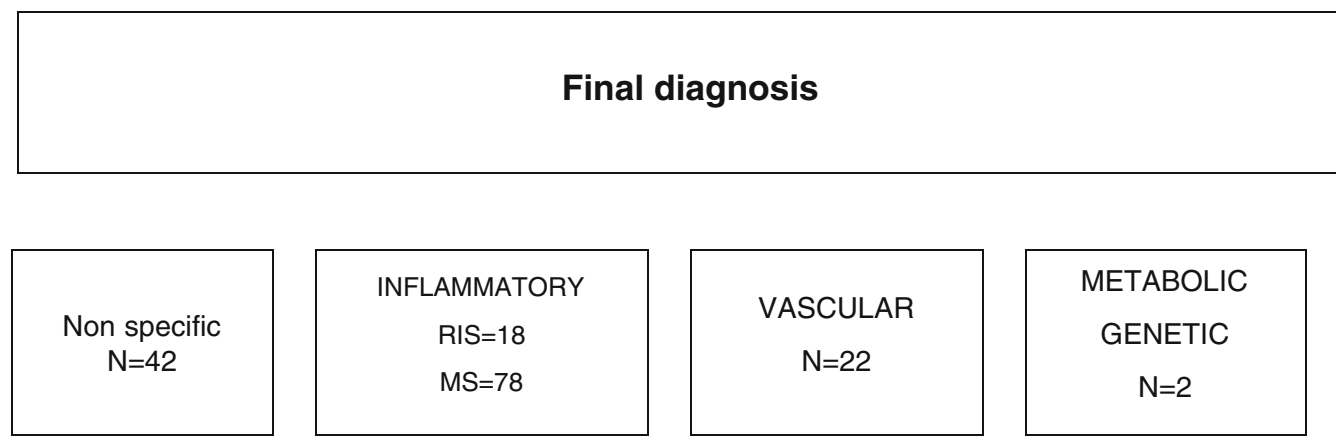

$\mathrm{CSF}=$ cerebrospinal fluid, $\mathrm{MS}=$ multiple sclerosis, VEP=visual evoked potential

Fig. 1 Methodical process of files from radiologically isolated syndrome suspected patients

$256 \times 192 ; 64$ slices; Axial Flair (FSE IR):

$\mathrm{TE}=157.5 ; \mathrm{TR}=10004 ; \mathrm{TI}=2,200 ; \mathrm{NEX}=1$;

FOV: $240 \times 240 \mathrm{~mm} ; 4.0 \mathrm{~mm} ; 256 \times 192 ; 28$

slices; Gadolinium injection $0.1 \mathrm{ml} / \mathrm{kg}$; Axial

T1 post-Gd (FSE-XL): $\quad \mathrm{TR}=480 ; \quad \mathrm{TE}=7.3$;

$\mathrm{NEX}=4 ; \quad$ FOV: $240 \times 180 \mathrm{~mm} ; \quad 2.0 \mathrm{~mm}$; $256 \times 192 ; 64$ slices.
All charts were reviewed at 2 years to confirm the final diagnosis, if possible.

\section{Diagnosis Proposal and Follow-up}

After a review of the patients' personal history, if symptoms suggestive of an inflammatory 
disease were found independently of the present MRI motive and MRI criteria of DS and DT were fulfilled, the patient was diagnosed with MS. Patients with possible RIS according to DS criteria but with a history of clinical symptom compatible with a first clinical event were classified as clinically isolated syndrome (CIS).

For the other recruited patients with nonspecific symptoms, a diagnosis was finally proposed on the combination of clinical, laboratory and radiological data.

\section{Compliance with Ethics}

All procedures followed were in accordance with the ethical standards of the responsible committee on human experimentation (institutional and national) and with the Helsinki Declaration of 1975 , as revised in 2000 and 2008. Informed consent was not required because all screenings were included in the standard clinical practice. The analysis in this article is based on previously conducted studies and does not involve any new studies of human or animal subjects performed by any of the authors.

\section{RESULTS}

Patient characteristics were: 165 women and 55 men, mean age: 42.7 years old (23-59). Patients were categorized as to whether they had symptoms and signs possibly related to $\mathrm{T} 2$ hypersignals (paraesthesia, vertigo, gait control), or unlikely to be specifically related to demyelination (isolated fatigue, headaches, trauma, endocrinopathy). After an extensive discussion, typical symptoms of demyelination, either optic neuritis or myelitis, evoking a CIS were found in $22 \%$ of
Table 1 Repartition of suspected radiologically isolated syndrome patients

\begin{tabular}{lll}
\hline MRI motive & N (\%) & $\begin{array}{l}\text { Clinical history } \\
(\%)\end{array}$ \\
\hline Headaches & $87(39)$ & 2 \\
$\begin{array}{l}\text { Atypical Sensitive } \\
\text { symptoms }\end{array}$ & $26(12)$ & 15 \\
Mood disorders & $22(10)$ & 3 \\
Visual & $20(9)$ & 11 \\
Fatigue & $17(8)$ & 1 \\
Hormonal screening & $13(6)$ & 2 \\
Vertigo & $13(6)$ & 2 \\
Trauma & $11(5)$ & 1.5 \\
Dummy run & $11(5)$ & 1.5 \\
\hline
\end{tabular}

patients with T2 hypersignals suggestive of RIS. These symptoms were not the MRI motive but the patient was then excluded.

The major symptom motivating brain MRI was headaches (39\%), sensitive atypical manifestations (12\%), mood disorders (10\%), transient visual symptoms (9\%), fatigue $(8 \%)$, hormonal screening $(6 \%)$, vertigo $(6 \%)$, cranial trauma (5\%), dummy run for clinical study (5\%) (Table 1).

After a structured analysis of T2 hyperintensities, the suspected diagnosis was: inflammatory $45 \%$, vascular 33\%, nonpathological $19 \%$, genetic $2 \%$, and metabolic $1 \%$. Extensive screening confirmed the proposed diagnosis in $97 \%$ of cases. From the 6- and 12-month visits, diagnosis was confirmed and only three cases remained unresolved. Among the 220 patients, only 78 (35.4\%) fulfilled the 2010 McDonald criteria, and 18 (8\%) were categorized as RIS (Table 2).

DS with MS/MRI criteria applied to the first brain MRI were fulfilled in $85 \%$ of MS, for $34.6 \%$ of the vascular patients, and for 0 of the nonspecific hyperT2 (Table 3). 38\% of patients 
Table 2 Comparison between the suspected and final diagnosis

\begin{tabular}{llll}
\hline Suspected diagnosis & N (\%) & Relevant Clinical history & Final diagnosis \\
\hline Inflammatory & $99(45 \%)$ & $49(22 \%)$ & RIS. 18 (8\%) \\
& & & MS. 78 (35\%) \\
& & Other inflammatory diseases. 4 (2\%) \\
Vasculopathy & $73(33 \%)$ & $22(10 \%)$ & $37(17 \%)$ \\
Non-pathological & $42(19 \%)$ & - & $68(31 \%)$ \\
Genetic or metabolic & $6(3 \%)$ & $4(2 \%)$ & $2(1 \%)$ \\
\hline
\end{tabular}

9 (4\%) patients had unexplained T2 abnormalities after 2 years of follow-up

$M S$ multiple sclerosis, RIS radiologically isolated syndrome

underwent initial spinal MRI. Among those who had T2 hyper intensities compatible with MS, 33\% of them had spinal T2 lesions.

Regarding the patients who had a final diagnosis of demyelinating disease, all MS/ MRI criteria have the same sensibility (Table 2) [2, 13]. The association of 2 DS criteria, a positive CSF and DT on MRI was also sensitive and specific. None of the patients with vascular diseases or nonpathological T2 hypersignals had MRI criteria and a positive CSF. DT criteria were present for $82.7 \%$ of MS patients and $36 \%$ of RIS patients but none of the vascular or non-pathological T2 abnormalities.

At 6 months, $35.4 \%$ of patients had finally been diagnosed as inflammatory disease with DT and DS. On the 24-month visit, diagnosis was confirmed and only three cases remained unresolved. For cases other than RIS or MS, diagnosis was confirmed with the paraclinical screening and identification of risk factors such as cardiovascular risks, metabolic abnormalities, and genetic diagnosis (one case of fragile $\mathrm{X}$-associated tremor/ataxia syndrome). The 2-year diagnostic distribution was: RIS $8 \%$, MS $35 \%$, other inflammatory diseases $2 \%$, ischemic vascular disease $17 \%$, non-pathological $31 \%$, genetic $1 \%$, and unknown $4 \%$.

\section{DISCUSSION}

Brain MRI is the most sensitive paraclinical diagnostic test for MS, but white matter abnormalities are also known to be present in many other circumstances [1, 14-16]. They have been reported in $40-95 \%$ of patients with other neurological diseases [8-10] and even in $44 \%$ of elderly asymptomatic patients [13]. Failure to consider the aspect of T2 hypersignals can induce misdiagnosis in some patients fulfilling the criteria of DT and DS. In these cases, proposing specific treatments that are available now can be risky.

Among our 220 suspected MS patients, 43\% were diagnosed with demyelinating disease and $22 \%$ with clinical MS, due to history of symptoms suggestive of a clinical event, acute or progressive. With an extensive questionnaire, neurologists can detect previous neurological event that patients failed to report. This contrasts with another study where $11 \%$ of 104 patients had suspected MS based on MRI [17]. Recently, the PEDIAS study revealed that $44 \%$ of patients with a typical CIS had a neurological history suggestive of a previous demyelinating event [18].

Other published studies showed that in the vast majority of patients who were referred with 
Table 3 Data (\% of patients) on fulfilled MRI criteria according to the final diagnosis

\begin{tabular}{|c|c|c|c|c|c|}
\hline MRI criteria (\%) & MS (\%) & $\begin{array}{l}\text { Other inflammatory } \\
\text { diseases (connectivitis) (\%) }\end{array}$ & Vasculopathy (\%) & RIS (\%) & $\begin{array}{l}\text { Non- } \\
\text { pathological } \\
(\%)\end{array}$ \\
\hline 3 DS criteria BARKHOF & 85 & 20 & 34.6 & 100 & 0 \\
\hline PATY & 90 & 50 & 72.3 & 71.4 & 33.3 \\
\hline FAZEKAS & 90 & 20 & 3.9 & 14.3 & 0 \\
\hline SWANTON & 90 & 40 & 42.3 & 88.6 & 6.7 \\
\hline Non-specific T2 hypersignals & 5 & $\mathbf{0}$ & 7.7 & 28.6 & 86.7 \\
\hline 2 DS criteria + CSF abnormalities & 57.8 & 30 & 0 & 42.3 & 0 \\
\hline DT MRI only (new T2 or gadolinium + ) & 82.7 & 0 & 0 & 36 & 0 \\
\hline Association of $2 \mathrm{HS}+\mathrm{CSF}$ and DT on MRI & 90 & 30 & $\mathbf{0}$ & 44 & $\mathbf{0}$ \\
\hline
\end{tabular}

$D S$ dissemination in space, $D T$ dissemination in time, $H S$ hypersignal, $M S$ relapsing remitting multiple sclerosis, CSF cerebrospinal spinal fluid

suspected demyelinating disease, but in whom no diagnosis could be made at the time of referral, no neurological diagnosis was made during an average follow-up of 7 years $[8,9]$. Finally, diagnostic uncertainty remains for a significant number of patients. For instance, the manifestations of a patient with non-specific, multifocal symptoms not typical of a CIS may still be wrongly diagnosed as possible MS. Correspondingly, potentially confirmatory MRI and other test results may be normal or nonspecifically abnormal. In these cases, definite diagnosis is not possible and follow-up is required.

In our cohort, the majority of T2 lesions diagnosed on MRI as possible MS were nonspecific lesions. The medical history and clinical analyses in MS diagnosis are very important [19]. In most patients in whom ultimately another diagnosis was made, it was found that at initial clinical presentation a diagnosis of MS could be rejected based on the association of medical history and MRI findings. Specific symptoms or signs pointing to the disease were not recognized.

With the extending accessibility of MRI, RIS is frequently overdiagnosed, either because medical history examination reveals a suggestive clinical event or the structured MRI analysis rejects the diagnosis of an inflammatory disease. Among our 220 patients, only 18 were finally diagnosed as RIS, others having either neurological symptoms history or T2 lesions nonsuggestive of MS. Even if incidental brain T2 lesions are frequent, asymptomatic patients with hypersignals and/or gadolinium enhancement fulfilling Barkhof/Tintoré MRI criteria and DT and DS are more rare [12, 20-24]. DT criteria were present for $82.7 \%$ of MS patients and $36 \%$ of RIS patients but none of the vascular or nonpathological T2 abnormalities.

In these published cohorts, $80 \%$ of patients with a RIS already fulfilled diagnostic criteria before the seminal event. In European or North American observational studies, the authors have found that up to $30-45 \%$ of patients with RIS will present clinical progression. [12, 21] The median time to clinical conversion differs between studies being 2.3 years for the series of French patients and 5.4 years for the American. Most patients who develop clinical symptoms had prior radiological progression. The presence of asymptomatic lesions in the 
cervical cord provides an increased risk of progression, either to relapsing or to progressive MS.

In longitudinal studies, it has been shown that $88 \%$ of patients with brain $\mathrm{T} 2$ abnormalities developed MS and even if new lesions can be clinically silent, the ratio of MRI activity to clinical relapse is approximately $7-10 \%$ [4, 6, 15]. Nevertheless, before the clinical threshold, other inflammatory diseases might always be suspected. Many inflammatory or infectious disorders (e.g., systemic lupus erythematosus, sarcoidosis and neuroborreliosis) that are commonly part of the differential diagnosis can be investigated by blood and CSF analyses [10]. Patients with migraine are also frequently diagnosed with $\mathrm{T} 2$ abnormalities and may be over diagnosed as RIS [25-27].

When applied intentionally, MRI and McDonald criteria are very specific and sensitive, providing their application is limited to MRI abnormalities suggestive of MS, after exclusion of 'red flags'. In this study's cohort, there were neither false positives nor false negatives for other pathologies than MS.

The paraclinical screening, for which there is no international consensus in the literature, is not mandatory for diagnosis of MS. Nevertheless, it can be very useful to diagnose DS or exclude other diagnoses [28-31]. Even if this population is clearly different from that seen in general neurological clinics and is biased towards diagnostic uncertainty, a lot of investigations could be spared if the diagnostic criteria are correctly applied.

\section{CONCLUSION}

Patients and their physicians could be more at ease if radiological reports were not so affirmative concerning the MS diagnosis without considering the clinical aspects. Nevertheless, some tests are suggestive of a CNS inflammatory disease, reinforcing diagnosis and bringing in another element for prognosis, thereby guiding treatment. Given the benefits of early treatment in MS, but also their further implications, diagnosis as soon as possible is certainly essential.

Despite advancements in the characterization of RIS subjects and in the understanding of risk factors for initial symptom development, the natural course of such cases and risk profiles for a seminal neurological event, from prospectively acquired data, remain unclear.

\section{ACKNOWLEDGMENTS}

There is no funding to declare for this work. All named authors meet the ICMJE criteria for authorship for this manuscript, take responsibility for the integrity of the work as a whole, and have given final approval to the version to be published.

Conflicts of interest. Christine Lebrun received honoraria for scientific boards from Bayer, Novartis, Teva, Almirall, Merck, Biogen Idec, Genzyme. Mikael Cohen received honoraria for scientific boards from Bayer, Novartis, Teva, Genzyme, Merck, Biogen Idec. Annabelle Chaussenot, Lydiane Mondot and Stephane Chanalet have no conflict of interest to declare.

Ethical standard. All procedures followed were in accordance with the ethical standards of the responsible committee on human experimentation (institutional and national) and with the Helsinki Declaration of 1975 , as 
revised in 2000 and 2008. Informed consent was not required because all screenings were included in the standard clinical practice. The analysis in this article is based on previously conducted studies, and does not involve any new studies of human or animal subjects performed by any of the authors.

Open Access. This article is distributed under the terms of the Creative Commons Attribution Noncommercial License which permits any noncommercial use, distribution, and reproduction in any medium, provided the original author(s) and the source are credited.

\section{REFERENCES}

1. McDonald WI, Compston A, Edan G, et al. Recommended diagnostic criteria for multiple sclerosis: guidelines from the International Panel on the Diagnosis of Multiple Sclerosis. Ann Neurol. 2001;50:121-7.

2. Barkhof F, Filippi M, Miller DH, et al. Comparison of MRI criteria at first presentation to predict conversion to clinically definite multiple sclerosis. Brain. 1997;120:2059-69.

3. Polman $\mathrm{CH}$, Reingold SC, Banwell B, et al. Diagnostic criteria for multiple sclerosis: 2010 Revisions to the McDonald criteria. Ann Neurol. 2011;69(2):292-302.

4. Tintoré M, Rovira A, Martinez M, et al. Isolated demyelinating syndromes: comparison of different MR imaging criteria to predict conversion to clinically definite multiple sclerosis. Am J Neuroradiol. 2000;21:702-6.

5. Tintoré M, Rovira A, Rio J, et al. New diagnostic criteria for multiple sclerosis: application in first demyelinating episode. Neurology. 2003;60:27-30.

6. Frohman EM, Goodin DS, Calabresi PA, et al. The utility of MRI in suspected MS: report of the Therapeutics and Technology Assessment Subcommittee of the American Academy of Neurology. Neurology. 2003;61:602-11.

7. Swanton JK, Rovira A, Tintoré M, et al. MRI criteria for multiple sclerosis in patients presenting with clinically isolated syndromes: a multicentre retrospective study. Lancet Neurol. 2007;6:677-86.
8. Nielsen JM, Korteweg $\mathrm{T}$, Barkhof $\mathrm{F}$, et al. Overdiagnosis of multiple sclerosis and magnetic resonance criteria. Ann Neurol. 2005;58:781-3.

9. Nielsen JM, Uitdehaag BM, Polman CH. Long-term follow-up of suspected though unconfirmed MS. Mult Scler. 2008;14(7):985-7 Epub 2008 Jun 23.

10. Charil A, Yousry TA, Rovaris M, et al. MRI and the diagnosis of multiple sclerosis: expanding the concept of no better explanation. Lancet Neurol. 2006;5:841-52.

11. Korteweg $\mathrm{T}$, Tintoré $\mathrm{M}$, Uitdehaag $\mathrm{B}$, et al. MRI criteria for dissemination in space in patients with clinically isolated syndromes: a multicentre followup study. Lancet Neurol. 2006;5:221-7.

12. Okuda DT, Mowry EM, Beheshtian A, et al. Incidental MRI anomalies suggestive of multiple sclerosis: the radiologically isolated syndrome. Neurology. 2009;72(9):800-5.

13. Fazekas F, Offenbacher H, Fuchs S, et al. Criteria for an increased specificity of MRI interpretation in elderly subjects with suspected multiple sclerosis. Neurology. 1988;38:1822-5.

14. Paty DW, Oger JJ, Kastrukoff LF, et al. MRI in the diagnosis of MS: a prospective study with comparison of clinical evaluation, evoked potentials, oligoclonal banding, and CT. Neurology. 1988;38:180-5.

15. Miller DH, Filippi M, Fazekas F, et al. Role of magnetic resonance imaging within diagnostic criteria for multiple sclerosis. Ann Neurol. 2004;56:273-8.

16. Filippini G, Comi GC, Cosi V, et al. Sensitivities and predictive values of paraclinical tests for diagnosing multiple sclerosis. J Neurol. 1994;241:132-7.

17. Carmosino MJ, Brousseau KM, Arciniegas DB, Corboy JR. Initial evaluations for multiple sclerosis in a university multiple sclerosis center: outcomes and role of magnetic resonance imaging in referral. Arch Neurol. 2005;62:585-90.

18. Gout $\mathrm{O}$, Moreau T, Debouverie M, Allouche S, on behalf of the Club Francophone de la sclérose en plaques. Pedias: pilot multicentre observational study aiming to standardize diagnosis of an inflammatory neurological event leading patient to consult for the first time. Mult Scler. 2007;13: P370.

19. Mushlin AI, Mooney C, Grow V, Phelps CE. The value of diagnostic information to patients with suspected multiple sclerosis. Rochester Toronto MRI Study Group. Arch Neurol. 1994;51:67-72. 
20. Lebrun C, Bensa C, Debouverie $M$, et al. Unexpected multiple sclerosis: follow-up of 30 patients with magnetic resonance imaging and clinical conversion profile. J Neurol Neurosurg Psychiatry. 2008;79(2):195-8.

21. Lebrun C, Bensa C, Debouverie M, et al. Association between clinical conversion to multiple sclerosis in radiologically isolated syndrome and magnetic resonance imaging, cerebrospinal fluid, and visual evoked potential: follow-up of 70 patients. Arch Neurol. 2009;66(7):841-6.

22. Okuda DT, Siva A, Kantarci O, et al. Radiologically isolated syndrome: 5-year risk for an initial clinical event. PLOS ONE. 2014;9(3):e90509.

23. Granberg T, Martola J, Aspelin P, et al. Radiologically isolated syndrome: an uncommon finding at a university clinic in a high-prevalence region for multiple sclerosis. BMJ Open. 2013;3:e003531. doi:10.1136/bmjopen-2013-003531.

24. Nakamura $M$, Morris $M$, Cerghet $M$, et al. Longitudinal follow-up of a cohort of patients with incidental abnormal magnetic resonance imaging findings at presentation and their risk of developing multiple sclerosis. Int J MS Care. 2014;16:111-5.

25. Cooney BS, Grossman RI, Farber RE, Goin JE, Galetta SL. Frequency of magnetic resonance imaging abnormalities in patients with migraine. Headache. 1996;36:616-21.
26. Pavese N, Canapicchi R, Nuti A, et al. White matter MRI hyperintensities in a hundred and twenty-nine consecutive migraine patients. Cephalalgia. 1994;14(5):342-5.

27. Liu S, Kullnat J, Bourdette D, et al. Prevalence of brain magnetic resonance imaging meeting Barkhof and McDonald criteria for dissemination in space among headache patients. Mult Scler. 2013;19(8):1101-5.

28. Freedman MS, Thompson EJ, Deisenhammer F, et al. Recommended standard of cerebrospinal fluid analysis in the diagnosis of multiple sclerosis: a consensus statement. Arch Neurol. 2005;62(6):865-70.

29. Gronseth GS, Ashman EJ. Practice parameter: the usefulness of evoked potentials in identifying clinically silent lesions in patients with suspected multiple sclerosis (an evidence-based review). Report of the Quality Standards Subcommittee of the American Academy of Neurology. Neurology. 2000; 9; 54(9): 1720-1725.

30. Tintoré M, Rovira A, Río J, et al. Do oligoclonal bands add information to MRI in first attacks of multiple sclerosis? Neurology. 2008;70(13 Pt 2):1079-83.

31. Villar LM, García-Barragán N, Sádaba MC, et al. Accuracy of CSF and MRI criteria for dissemination in space in the diagnosis of multiple sclerosis. J Neurol Sci. 2008; 15; 266(1-2): 34-7. 